\title{
Toll story
}

$\mathbf{P}$ our se défendre contre les micro-organismes, les vertébrés disposent d'un système immunitaire spécifique qui s'adapte à chaque agresseur. Cette immunité adaptative, fondée sur une prolifération clonale de lymphocytes et la synthèse d'anticorps, nécessite plusieurs jours pour se développer. En outre, comme le sait tout biologiste ayant été amené à obtenir des anticorps chez un animal, l'injection d'un antigène pur ne permet pas d'obtenir une réponse significative et il convient de lui adjoindre un adjuvant constitué d'un mélange d'extraits microbiens. En fait, chez tous les vertébrés, l'invasion par des micro-organismes est initialement combattue par des mécanismes de défense innée qui préexistent chez tous les individus et sont activés dans les minutes qui suivent l'infection. Les adjuvants qu'il est nécessaire d'injecter avec l'antigène pour obtenir une bonne réponse ont précisément pour fonction d'activer ce bras inné de la réponse immunitaire [1].

Quoique les travaux de Metchnikoff à l'Institut Pasteur aient ouvert la voie dès la fin du XIX ${ }^{e}$ siècle, ce n'est que dans les dix dernières années que l'on a commencé à comprendre l'importance de ce système immunitaire inné qui, outre sa capacité à neutraliser les micro-organismes, déclenche et oriente la réponse immunitaire spécifique. Les macrophages, cellules effectrices de la réponse immunitaire innée des mammifères, sont activés par certaines des molécules décorant spécifiquement les différents types de pathogènes comme les lipopolysaccharides (LPS) des bactéries à Gram négatif, le peptidoglycane des parois des bactéries à Gram positif et les mannanes de la paroi des champignons. Charles Janeway a proposé d'appeler PAMP (pathogen associated molecular pattern) ces activateurs universels [2]. Les macrophages activés répondent par la phagocytose des bactéries et la production de cytokines comme l'interleukine (IL) -1, le TNF $\alpha$ (tumor necrosis facteur $\alpha$ ) et l'IL-6 qui déclenchent la réponse inflammatoire et activent les lymphocytes du bras adaptatif du système immunitaire. Bien qu'extrêmement efficace au sein des tissus, cette activation des macrophages par les PAMP peut avoir des conséquences dramatiques lorsqu'elle a lieu dans la circulation. On assiste alors à une activation des macrophages dans le foie et la rate, et à un relargage systémique de TNF $\alpha$ dans le sang. La perméabilité accrue des vaisseaux sanguins qui en résulte conduit à de nombreux événements de coagulation intravasculaire qui causent une défaillance multiviscérale et la mort par choc septique. Malgré la grande importance clinique de ces chocs septiques, qui tuent chaque année des dizaines de milliers de personnes dans le monde et ont valu au LPS le nom d'endotoxine, les récepteurs capables d'activer les macrophages en réponse aux PAMP sont longtemps restés élusifs.

Des travaux récents ont montré l'existence d'une réponse innée dans des groupes aussi distants que les mammifères et les insectes, suggérant une origine évolutive commune. Ainsi, le système immunitaire inné constituerait un mécanisme de défense commun à tous les animaux multicellulaires et le système immunitaire spécifique, qui n'est apparu que tardivement à partir des sélaciens, se serait superposé à ce mécanisme de défense très ancien [1]. Génétiquement bien connue, la drosophile (ou mouche du vinaigre) a constitué un modèle de choix pour l'étude des réactions de défense innée contre les micro-organismes. La découverte de l'importance du récepteur Toll dans la réponse antifongique de cet insecte a suggéré la recherche d'homologues chez les mammifères et a mené à la décou- verte d'un composant central du récepteur du LPS dans les macrophages des mammifères.

\section{La découverte de Toll}

Au début des années 1980, Christiane Nüsslein-Volhard et Éric Wieschaus entreprirent les premiers cribles génétiques dans le dessein d'isoler des mutants du développement embryonnaire précoce chez la drosophile, travaux qui leur vaudront le prix Nobel de médecine en 1995. Au cours d'un de ces cribles, E. Wieschaus découvrit une lignée de drosophiles dont les femelles pondaient des œufs à développement embryonnaire avorté. Observant l'aspect de la cuticule de ces embryons et réalisant que la mutation avait un effet sur la détermination de l'axe dorso-ventral inverse de celui qu'elle observait pour ses propres mutants, C. Nüsslein-Volhard s'exclama "Toll» (ce qui veut dire, en allemand vernaculaire, «fantastique» ou «épatant»). Par la suite, le récepteur Toll s'est avéré être le pivot d'une voie de transduction du signal, la voie Toll, comportant 12 gènes et établissant la polarité dorso-ventrale et l'identité tissulaire de l'embryon de drosophile [3] (figure 1).

Une cascade de protéases à sérine est activée dans l'espace périvitellin du côté ventral de l'embryon par un signal en provenance des cellules folliculaires entourant l'ovocyte au cours de sa maturation. La dernière protéase de cette cascade, Easter, clive le précurseur de la protéine Spaetzle, qui possède une structure en nœud de cystéine analogue à celle de certains facteurs de croissance comme le NGF (nerve growth factor) ou le VEGF (vascular endothelial growth factor) [4]. Ce ligand se fixe alors vraisemblablement sur le récepteur transmembranaire Toll qui active une voie de transduction intracellulaire aboutissant à la phosphorylation 


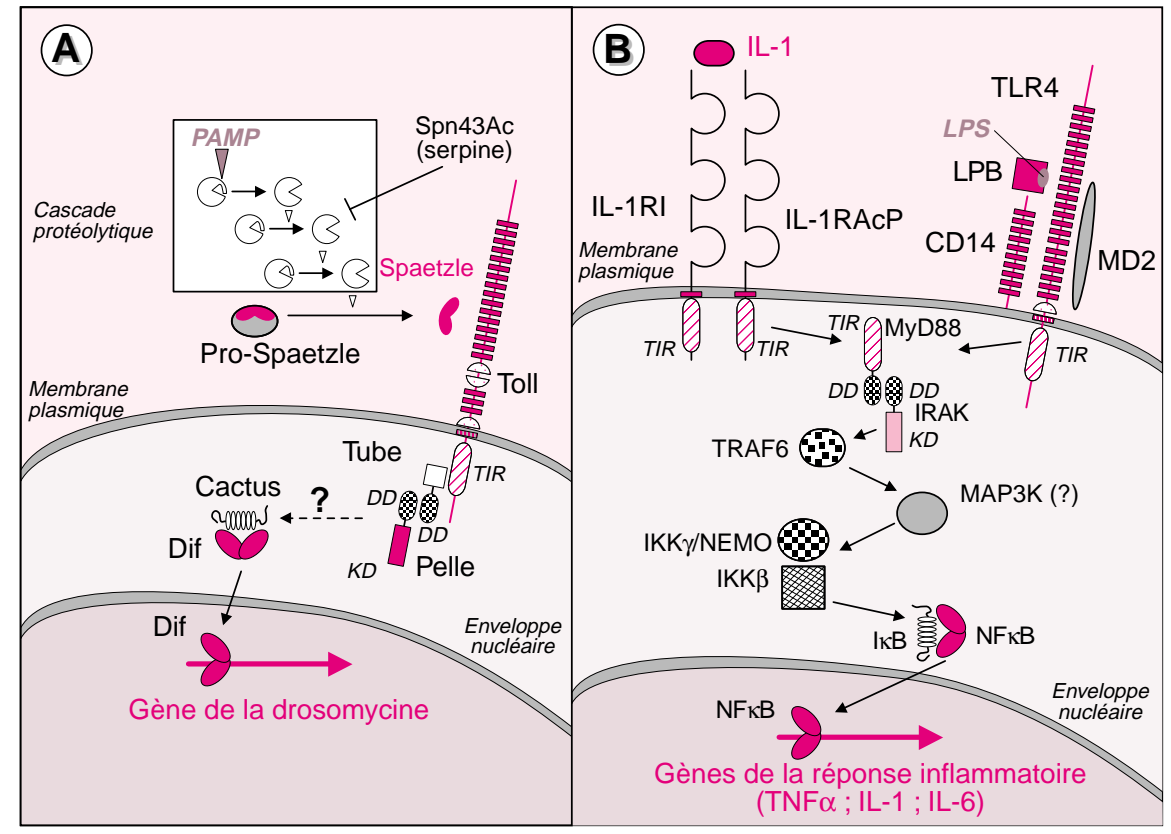

Figure 1. Les voies de signalisation contrôlant la réponse antifongique chez la drosophile $(A)$ et la réponse inflammatoire chez les mammifères (B). A. $\dot{A}$ la suite $d^{\prime}$ une infection fongique, on pense qu'un déterminant moléculaire (pathogen associated molecular pattern: PAMP) de la paroi des champignons (un mannane par exemple) est reconnu par un zymogène et que cette interaction active une cascade protéolytique. Une de ces protéases clive le précurseur de la cytokine Spaetzle qui va activer le récepteur Toll. Toll induit la phosphorylation et la dégradation de l'inhibiteur cactus par l'intermédiaire de tube et de pelle. Ceci permet la translocation nucléaire du facteur de transcription Dif [20], et l'induction du gène codant pour le peptide antifongique Drosomycine. B. Au cours de la réponse inflammatoire des mammifères, le récepteur de I'IL-1 (IL 1-R) et I'homologue de Toll, TLR4, interagissent avec MyD88 par leur domaine TIR conservé. IL 1-R est activé par son ligand et TLR4 par le LPS des bactéries à Gram négatif après sa fixation à la LBP (LPS binding protein) et à CD14. Ceci déclenche une cascade de transduction qui aboutit à la phosphorylation $d^{\prime} / \kappa B$ par la kinase IKK $\beta$ (pour revue, voir $[21,22])$. I $\kappa B$ est ensuite ubiquitinylé et dégradé, laissant $N F-\kappa B$ libre de migrer dans le noyau et d'activer la transcription. TIR: domaine d'homologie Toll/IL-1R; $D D$ (death domain) KD: domaine kinase (kinase domain). Les cytokines sont représentées en rouge et les molécules dérivées de la paroi des micro-organismes en bleu. Les répétitions riches en leucine et les motifs riches en cystéines des domaines extracellulaires des molécules Toll sont respectivement représentés par des rectangles et des demi-cercles. Les domaines immunoglobulines sont représentés par des boucles.

et à la dégradation de l'inhibiteur cactus, et à la translocation nucléaire du facteur de transcription Dorsal qui active ou réprime plusieurs gènes cibles.

\section{Toll et la réponse antifongique chez la drosophile}

Le système de défense de la drosophile contre les infections micro- sal était, à cette époque, la seule protéine de cette famille connue chez la mouche et son rôle dans l'établissement de l'axe dorso-ventral de l'embryon était bien établi. De plus, la voie de transduction qui conduit le signal de Toll jusqu'à Cactus s'est avérée structuralement similaire à la voie de signalisation qui active NF- $\kappa \mathrm{B}$ à partir du récepteur de l'IL-1 (IL-1R) au cours de la réponse immunitaire. On trouve ainsi, en aval du domaine intra-cytoplasmique conservé de Toll et IL-1R (ou domaine TIR, pour Toll, IL-1 receptor), des molécules adaptatrices à "Death Domain " comme Tube et MyD88, des kinases homologues comme Pelle et IRAK (m/s 1996, $\left.n^{\circ} 5, p .639\right)$, enfin les molécules inhibitrices de protéines Rel, Cactus et IאB [7] (figure 1B). Ceci nous conduisit à tester l'effet de mutants de la voie Toll sur la réponse immunitaire de la drosophile et à montrer qu'en l'absence de ce récepteur, les mouches succombent rapidement aux affections fongiques [8] (pour revue, voir [9]).

\section{Toll chez les mammifères}

Après s'être inspiré du modèle inflammatoire et de l'homologie entre les domaines de signalisation de Toll et du récepteur de l'IL-1, le modèle drosophile allait à son tour servir de guide aux immunologistes et aux médecins. C. Janeway et ses collaborateurs à l'Université de Yale aux États-Unis furent parmi les premiers à mesurer la portée des résultats obtenus dans le modèle drosophile et à rechercher des homologues de Toll chez les mammifères. La description par ce groupe en août 1997 [10] d'un homologue humain de Toll (depuis rebaptisé Toll like receptor TLR 4) capable d'activer NF- $\kappa \mathrm{B}$ et d'induire la synthèse de l'IL-1 et de l'IL-6, a déclenché une vague d'intérêt pour les récepteurs Toll. A cette date, six récepteurs de cette famille (TLR1-6) ont été décrits, et testés pour leur capacité à activer NF-אB. Deux autres gènes codant pour des récepteurs Toll sont présents dans les banques de données. Cependant, l'essentiel de l'intérêt a porté sur deux membres de cette famille, TLR2 et TLR4. Pendant que les travaux fondamentaux sur 
l'immunité de la drosophile mettaient en avant l'importance de Toll, plusieurs groupes dont celui de B. Beutler à Dallas et celui de D. Malo à Montréal entamaient le sprint final d'une course de 20 ans pour cloner et caractériser le locus lps. Cette mutation, identifiée dans les années 1970 dans les souches de souris C57BL/10ScCr et C3H/HeJ, rend les animaux extrêmement sensibles aux infections par les germes à Gram négatif [11]. Le groupe de Beutler séquença une région d'une mégabase dans laquelle il avait cartographié le locus mutant. L'examen de la séquence du gène TLR4, présent dans ce locus, mit en évidence une mutation ponctuelle donnant naissance à une protéine incapable d'activer NF$\kappa \mathrm{B}(\mathrm{C} 3 \mathrm{H} / \mathrm{HeJ})$ ou une délétion du gène entier (C57BL/10ScCr) ([12]; voir aussi [13]). Ces résultats ont récemment été confirmés chez l'homme, puisque des mutations dans le gène de TLR4 ont été retrouvées chez des sujets présentant une hypersensibilité au LPS dans les voies respiratoires [14]. Ces travaux indiquent que TLR4 est nécessaire pour l'activation de NF- $\kappa B$ par le LPS, et suggèrent que d'autres TLRs pourraient jouer un rôle analogue en réponse à d'autres PAMP. Cette hypothèse a maintenant été validée par la description du phénotype de souris chez lesquelles le gène TLR2 a été invalidé. Ces animaux sont défectifs pour la réponse au peptidoglycane, bien que leur réponse au LPS soit normale [15]. Il semble ainsi que différents membres de la famille Toll soient impliqués dans la détection de PAMP spécifiques de certains types de microorganismes. Curieusement cependant, TLR2 et TLR4 semblent activer les mêmes voies de transduction, et aucune réponse spécifique de l'un ou de l'autre de ces récepteurs n'a encore pu être mise en évidence.

\section{Le ligand des récepteurs Toll}

Si la conservation des voies de signalisation des récepteurs Toll chez les mammifères et les insectes ne fait pas de doute, les choses sont moins claires pour ce qui est de l'activation de ces récepteurs. Dans l'embryon de drosophile, il a été montré que le ligand de
Toll, Spaetzle, est clivé sous l'action d'une cascade de protéases, ellemême activée par la localisation ventrale d'un glycosaminoglycane dont les sucres sont sulfatés [16]. De façon surprenante, les protéases qui sont impliquées dans le clivage de Spaetzle chez l'embryon n'interviennent pas dans la réponse immunitaire. Cependant, Spaetzle est nécessaire pour l'activation de Toll pendant la réponse immunitaire, et une mutation provoquant une perte de fonction dans le gène d'un inhibiteur de protéase à sérine (une serpine) entraîne une activation constitutive de l'ensemble de la voie Toll [17]. Ceci suggère fortement que l'activation de Toll pendant la réponse immunitaire implique une cascade de protéases à sérine distincte de celle du développement embryonnaire.

Chez les mammifères, deux types de scénarios sont imaginables. Dans le premier, un mécanisme similaire à celui de la drosophile entraînerait l'activation de protéases à sérine sériques et l'activation d'un ligand de type Spaetzle. Dans le second, les PAMP et le LPS en particulier, pourraient être reconnus directement par les TLR. Des résultats récents obtenus par le groupe de D. Golenbock à Boston tendent à favoriser la seconde hypothèse. Ces chercheurs ont utilisé les propriétés pharmacologiques d'analogues du lipide A (la partie bioactive du LPS) pour démontrer que TLR4 est étroitement associé au LPS au sein du complexe récepteur sur les membranes des macrophages [18]. Il semble donc que l'activation de Toll chez la drosophile et de TLR4 dans les macrophages de mammifère se fasse par des mécanismes radicalement différents, impliquant une molécule de type cytokine chez la drosophile, et une reconnaissance directe du non-soi chez les mammifères.

\section{Conclusions}

Malgré les progrès spectaculaires de ces deux dernières années, il est clair que l'ère des récepteurs Toll ne fait que commencer et qu'il nous reste beaucoup à apprendre de ces molécules. Un rôle a pu être assigné au Toll original de drosophile ainsi qu'à TLR2 et TLR4 chez les mammifères.
Chez les plantes, plusieurs gènes de résistance aux maladies codent pour des facteurs intra-cytoplasmiques contenant un domaine TIR. Cette convergence de fonction chez des organismes multicellulaires aussi divers que les plantes, les insectes ou les mammifères suggère fortement que les protéines à domaine TIR descendent d'une molécule ancestrale dont la fonction primitive était l'activation des réactions de défense en réponse à un pathogène pourvu de motifs oligosaccharidiques "étrangers». Ce récepteur se serait ensuite dupliqué en divergeant pour s'adapter à la reconnaissance de nombreux PAMP différents. Ainsi, les différents TLR, en formant des hétérodimères, pourraient fournir des surfaces spécifiques pour de nombreux pathogènes différents. Parallèlement, chez certains insectes, le récepteur Toll aurait pu être secondairement coopté, à cause de sa fonction de reconnaissance des sucres, pour assurer la transmission d'un signal topologique et temporel entre la mère (les cellules folliculaires et leurs décorations glycosidiques) et l'embryon.

Chez la drosophile, il existe huit autres récepteurs apparentés à Toll [19], et le génome humain code pour au moins 10 TLR. Ces molécules sont-elles toutes impliquées dans la réponse immunitaire, et en réponse à quels signaux ? En particulier, existe-t-il des récepteurs Toll de drosophile activés par le LPS, comme chez les mammifères, et des TLRs de mammifères fonctionnant comme des récepteurs de cytokine ? L'analyse de drosophiles mutantes pour ces gènes et de souris chez lesquelles les gènes TLR ont été invalidés permettra de répondre à ces questions et de comprendre comment les récepteurs apparentés à Toll contrôlent la réponse immunitaire innée

\section{Jean-Marc Reichhart Jean-Luc Imler}

Université Louis Pasteur, IBMC, UPR 9022 du CNRS, "Réponse immunitaire et développement chez les insectes", 15, rue René-Descartes, 67084 Strasbourg Cedex, France. 


\section{RÉFÉRENCES}

1. Hoffmann JA, Kafatos FC, Janeway CA, Ezekowitz RA. Phylogenetic perspectives in innate immunity. Science 1999; 284: 1313-8.

2. Janeway CA, Jr. How the immune system recognizes invaders. Sci Am 1993; sept. : 41-7.

3. Belvin MP, Anderson KV. A conserved signaling pathway: the Drosophila toll-dorsal pathway. Ann Rev Cell Dev Biol 1996; 12 : 393-416.

4. Mizuguchi K, Parker JS, Blundell TL, Gay NJ. Getting knotted: a model for the structure and activation of Spatzle. Trends Biochem Sci 1998; 23: 239-42.

5. Bulet P. Les peptides antimicrobiens de la drosophile. Med Sci 1999; 15 : 23-9.

6. Imler JL, Hoffmann JA. Signaling mechanisms in the antimicrobial host defense of Drosophila. Curr Opin Microbiol 2000; 3: 1622.

7. Hoffmann JA, Reichhart JM. Drosophila immunity. Trends Cell Biol 1997; 7 : 309-16.

8. Lemaitre B, Nicolas E, Michaut L, Reichhart JM, Hoffmann JA. The dorsoventral regulatory gene cassette spatzle/Toll/cactus controls the potent antifungal response in Drosophila adults. Cell 1996; 86 : 973-83.

9. Lemaitre, B. La drosophile: un modèle pour l'étude de la réponse immunitaire innée. Med Sci 1999; 15: 15-22.
10. Medzhitov R, Preston-Hurlburt P, Janeway CA. Jr. A human homologue of the Drosophila Toll protein signals activation of adaptive immunity. Nature 1997; 388 : 394-7.

11. Watson J, Kelly K, Largen M, Taylor BA. The genetic mapping of a defective LPS response gene in $\mathrm{C} 3 \mathrm{H} / \mathrm{HeJ}$ mice. I Immunol 1978 ; 120 : 422-4.

12. Poltorak A, He X, Smirnova I, et al. Defective LPS signaling in $\mathrm{C} 3 \mathrm{H} / \mathrm{HeJ}$ and C57BL/10ScCr mice: mutations in Tlr4 gene. Science 1998; 282 : 2085-8. 13. Qureshi ST, Lariviere L, Leveque G,
Clermont S, Moore KJ, Gros P, Malo D. Endotoxin-tolerant mice have mutations in Toll-like receptor 4 (Tlr4). J Exp Med 1999; 189: 615-25.

14. Arbour NC, Lorenz E, Schutte BC, et al. TLR4 mutations are associated with endotoxin hyporesponsiveness in humans. Nat Genet 2000 ; 25 : 187-91.

15. Takeuchi O, Hoshino K, Kawai T, et al. Differential roles of TLR2 and TLR4 in recognition of gram-negative and grampositive bacterial cell wall components. Immunity 1999 ; 11 : 443-51.

16. Sen J, Goltz JS, Stevens L, Stein D. Spatially restricted expression of pipe in the Drosophila egg chamber defines embryonic dorsal-ventral polarity. Cell 1998; 95 : 471-81.

17. Levashina EA, Langley E, Green C, et al. Constitutive activation of toll-mediated antifungal defense in serpin-deficient Drosophila. Science 1999; 285: 1917-9.
18. Lien E, Means TK, Heine $\mathrm{H}$, et al. Tolllike receptor 4 imparts ligand-specific recognition of bacterial lipopolysaccharide. I Clin Invest 2000 ; 105 : 497-504.

19. Tauszig S, Jouanguy E, Hoffmann JA, Imler JL. Toll-related receptors and the control of antimicrobial peptide expression in Drosophila. Proc Natl Acad Sci USA 2000: 97: 10520-5.

20. Rutschmann S, Jung AC, Hetru C, Reichhart JM, Hoffmann JA, Ferrandon D. The Rel protein DIF mediates the antifungal but not the antibacterial host defense in Drosophila. Immunity 2000; 12: 569-80.

21. Israel A. The IKK complex: an integrator of all signals that activate NF-kappaB? Trends Cell Biol 2000; 10 : 129-33.

22. Karin M. The beginning of the end: IkappaB kinase (IKK) and NF-kappaB activation. J Biol Chem 1999; 274: 27339-42.

\section{TIRÉS À PART}

J.M. Reichhart. 